\title{
Sources and Clinical Applications of Mesenchymal Stem Cells State-of-the-art review
}

*Roberto Berebichez-Fridman ${ }^{1,2}$ and Pablo R. Montero-Olvera ${ }^{2}$

$$
\text { مصادر الخلايا الجذاعية الوسيطة واستخداماتا السريرية }
$$

روبرتو بيريبتشيز-فريدمان و بابلو رودريغو مونتيرو-أولفيرا

ABSTRACT: First discovered by Friedenstein in 1976, mesenchymal stem cells (MSCs) are adult stem cells found throughout the body that share a fixed set of characteristics. Discovered initially in the bone marrow, this cell source is considered the gold standard for clinical research, although various other sources-including adipose tissue, dental pulp, mobilised peripheral blood and birth-derived tissues-have since been identified. Although similar, MSCs derived from different sources possess distinct characteristics, advantages and disadvantages, including their differentiation potential and proliferation capacity, which influence their applicability. Hence, they may be used for specific clinical applications in the fields of regenerative medicine and tissue engineering. This review article summarises current knowledge regarding the various sources, characteristics and therapeutic applications of MSCs.

Keywords: Mesenchymal Stem Cells; Adult Stem Cells; Regenerative Medicine; Cell Differentiation; Tissue Engineering.

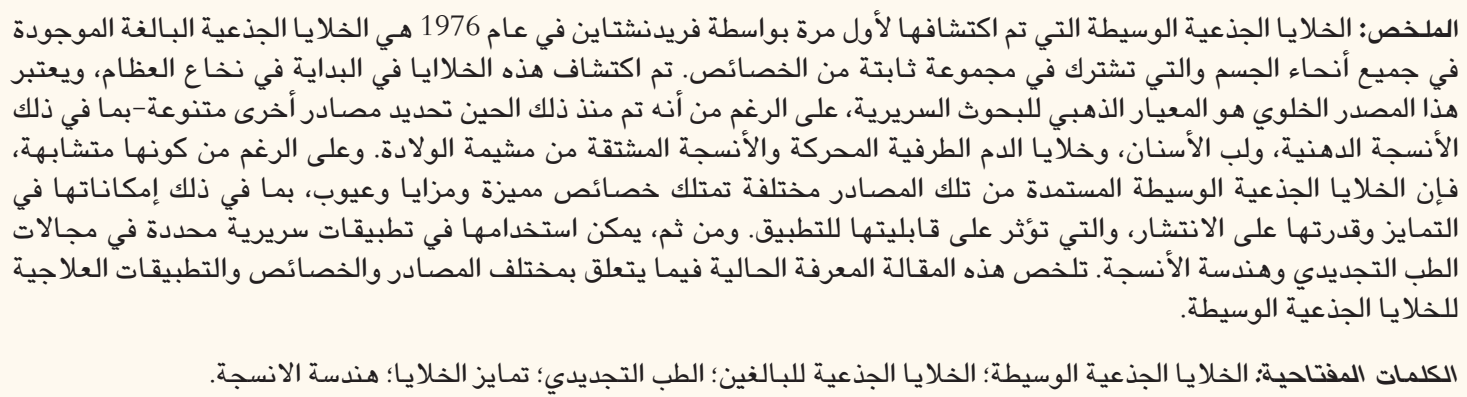

$\mathrm{S}$ TEM CELLS CAN BE DisTINGUished BASED ON their differentiation potential and source within the human body. Embryonic stem cells are totipotent, because they can form both embryonic and extraembryonic structures. ${ }^{1}$ Furthermore, embryonic stem cells can proliferate indefinitely under specific culture conditions and retain the ability to differentiate into cell types of the three embryonic germ layers. ${ }^{1,2}$ In contrast, adult stem cells are undifferentiated multipotent stem cells obtained from adult individuals and differentiate into the cell types that constitute their respective source tissues; accordingly, cells originating from neuronal tissue can differentiate into neurons, oligodendrocytes or astrocytes. This characteristic plasticity is an attribute of mesenchymal stem cells (MSCs), which are unspecialised cells with the ability to self-renew. ${ }^{3,4}$
Human MSCs are plastic-adherent cells that differentiate into cells that originate from the ectoderm and endoderm. ${ }^{3-5}$ Moreover, they can abandon their unspecialised or undifferentiated states and transform into other mesenchymal lineages. Thus, they can regenerate bone, cartilage and fat and even become endothelial cells, muscle cells or neurons under physiological and experimental conditions. ${ }^{3,4}$ While evidence suggests that MSCs are present in almost all human tissues, they were first isolated from mononuclear cells derived from bone marrow (BM). ${ }^{3,6}$

As MSCs are responsible for tissue repair, growth, wound healing and cell substitution resulting from physiological or pathological causes, they have various therapeutic applications such as in the treatment of central nervous system afflictions like spinal cord lesions. ${ }^{4}$ 
Moreover, because of their differentiation ability, MSCs have become the de facto model for regenerative medicine research. ${ }^{3,5,6}$ In the field of regenerative medicine, MSCs have several advantages over other types of stem cells. For example, from an ethical standpoint, the controversy that surrounds the procurement of embryonic stem cells is virtually nonexistent in the case of induced pluripotent stem cells or MSCs, although teratogenicity limits the widespread use of the former cell type. ${ }^{3,4,6}$

The objective of the current review was to highlight the available information regarding MSC sources and their potential applications in the treatment of a variety of diseases. However, in order to present a comprehensive overview of the possible clinical applications of MSCs, it is first necessary to understand their unique characteristics, including their differentiation potential, activity and therapeutic effects in various human systems and tissues.

\section{Differentiation Potential}

MSCs can differentiate into different cell types, although they require an array of differentiation factors. In an in vitro environment, the specific growth medium allows them to reach their osteogenic, chondrogenic or adipogenic potential. ${ }^{7,8}$ However, due to the use of different isolation and culture techniques, there are conflicting data regarding the specific characteristics used to define MSCs. Since these cells can be isolated from almost any tissue, it has been proposed that MSCs from various sources might not be similar enough to be grouped together under a single classification. ${ }^{6}$ However, this issue has been resolved following the establishment of universally accepted laboratory criteria allowing for the identification of common MSC characteristics. ${ }^{8}$

According to the International Society for Cellular Therapy, multipotent MSCs must meet the following three criteria: plastic adherence to culture flasks; expression of cluster of differentiation (CD)105, CD73 and CD90 and lack of expression of CD45, CD34, CD14/ CD11b, CD79 $\alpha / C D 19$ and human leukocyte antigen (HLA) class II by $\geq 95 \%$ and $\leq 2 \%$ of the cell population, respectively; and the ability to differentiate into osteoblasts, chondroblasts or adipocytes. ${ }^{8}$ In addition, under specific culture conditions, MSCs can differentiate into non-mesodermal lineages such as hepatocytes, neurons, pancreatic cells, cardiac muscle cells or astrocytes. ${ }^{9}$

\section{Sources and Clinical Applications}

Stem cells can be isolated from various sources in the human body, the selection of which should ideally be based on their logistical, practical and in vitro characteristics. Currently, the main sources of MSCs are BM and adipose tissue (AT). ${ }^{6}$ Although MSCs can hypothetically be obtained from almost any tissue within the human body, there are practical limitations concerning the difficulty and invasiveness of the procurement process and various donor characteristics. To select an adequate cell source, the practitioner must consider the difficulty of procuring the samples and the potential adverse effects of harvesting the cells on the donor. Obtaining BM-MSCs, for example, can result in pain, bleeding or infection, thus making harvesting MSCs from this source more problematic than harvesting cells from peripheral blood or surgical remnants such as AT or birth-derived tissues. ${ }^{9}$ Table 1 describes current sources of MSCs along with their characteristics, advantages, disadvantages and clinical applications. $^{7,9-30}$

\section{BONE MARROW}

Initially discovered in 1976 by Friedenstein, BM-MSCs were first described as undifferentiated MSCs in 1987.31,32 Subsequently, BM became the main source of multipotent stem cells. However, their procurement requires a highly invasive and painful procedure involving heavy use of anaesthesia; moreover, the cell yield, longevity and potential for differentiation diminishes with donor age. ${ }^{9}$ Compared with MSCs derived from other sources, BM-MSCs possess a longer duplication period, reach senescence earlier and constitute only $0.01-0.001 \%$ of nucleated marrow cells. ${ }^{9,10}$ Nevertheless, one advantage of BM-MSCs over other cell types is their relatively short culture time..$^{11,33}$

The role of inflammation in the pathogenesis of arterial hypertension, particularly in activating the sympathetic nervous system, has been recently identified in a study using a murine model. ${ }^{34}$ Penn et al. observed that BM transplantation from spontaneously hypertensive rats into normotensive rats resulted in a marked increase in blood pressure; however, treating the rats with minocycline, a second-generation semisynthetic tetracycline and microglial activation inhibitor with anti-inflammatory effects, reversed the hypertension. ${ }^{34}$ The underlying mechanism involves an increase in the production of inflammatory cells and cytokines which translates into a stronger neuroinflammatory response and increased activity of the sympathetic nervous system. The association between haematopoietic (i.e. BM-derived) stem cells and hypertension indicates that BM-MSC therapy may have potential applications in treating obesity, diabetes mellitus (DM) and hypertension. ${ }^{34}$

In a clinical context, attempts have been made to use BM-MSCs to alleviate heart failure, address cardio- 


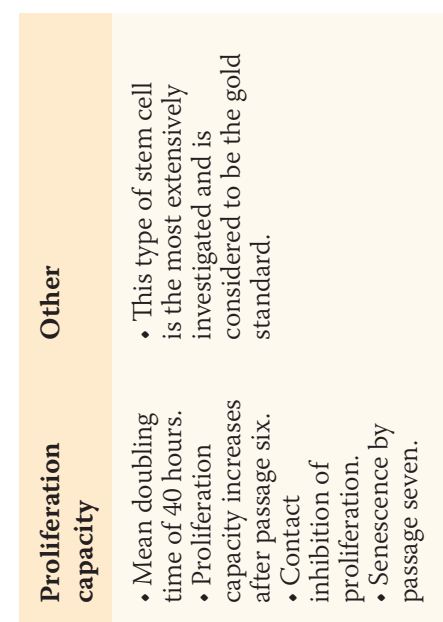

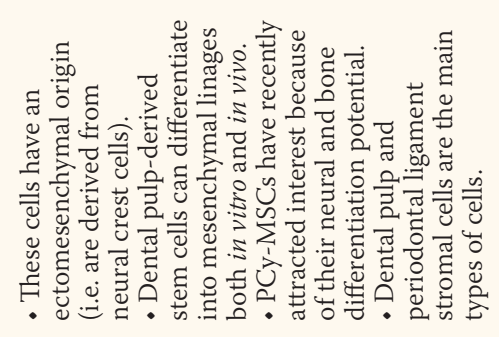
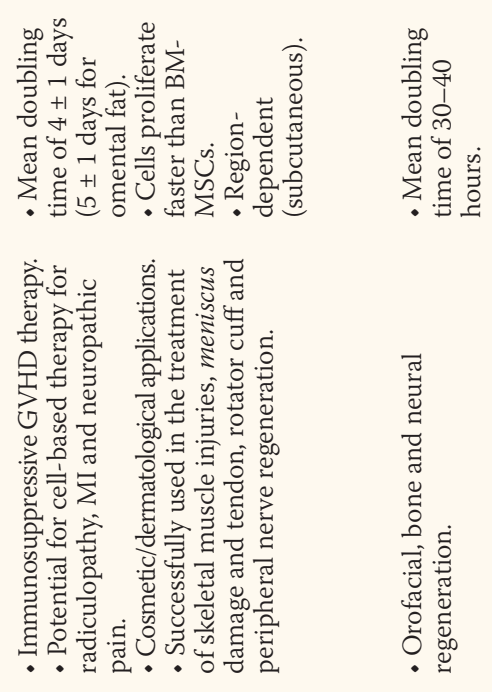

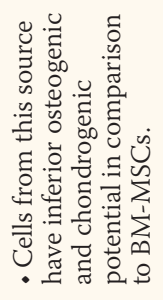

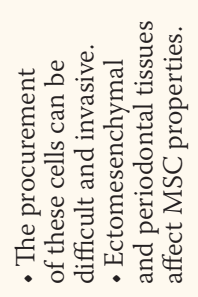

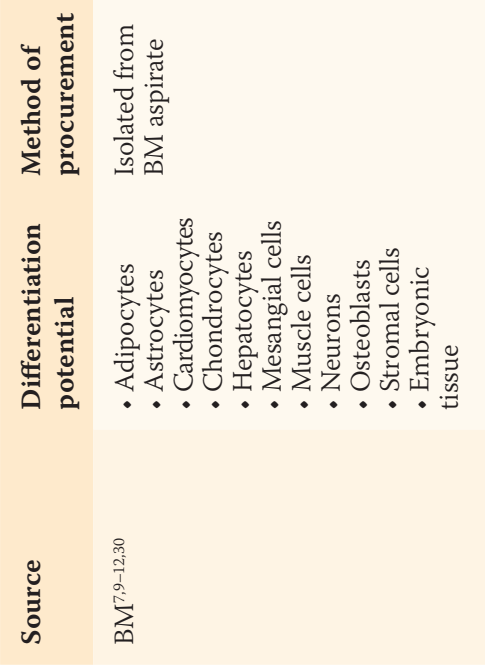
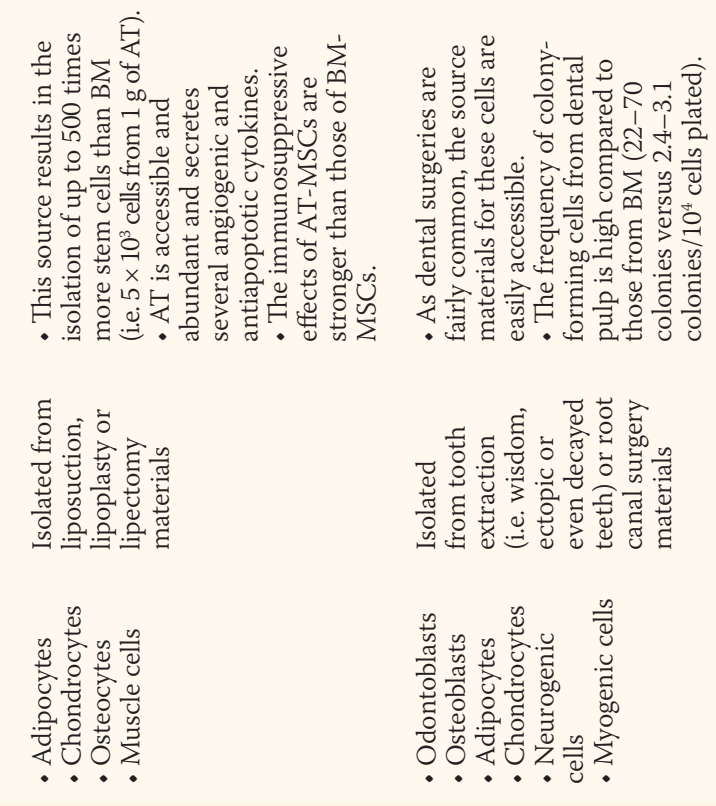


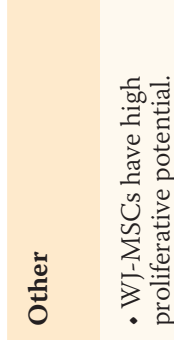

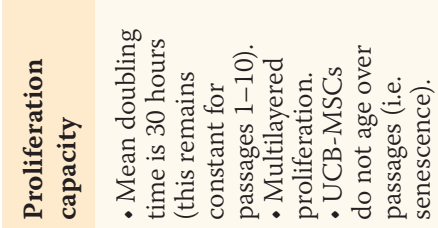

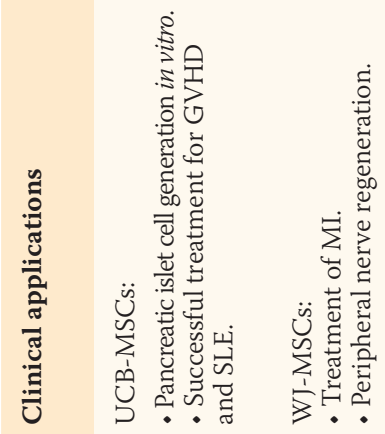

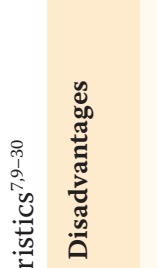
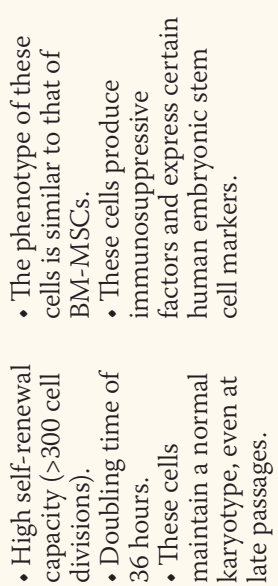

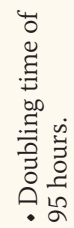
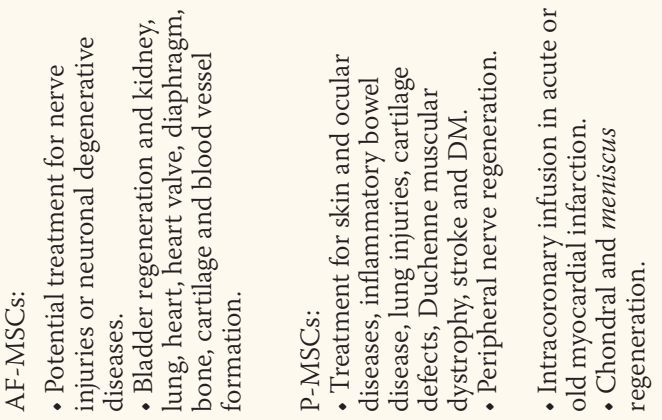

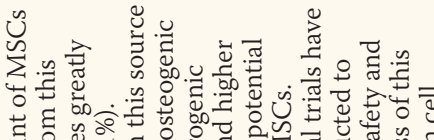

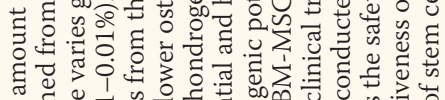

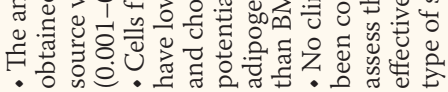

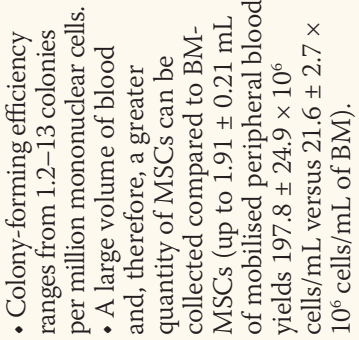

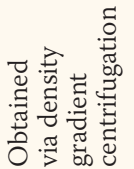
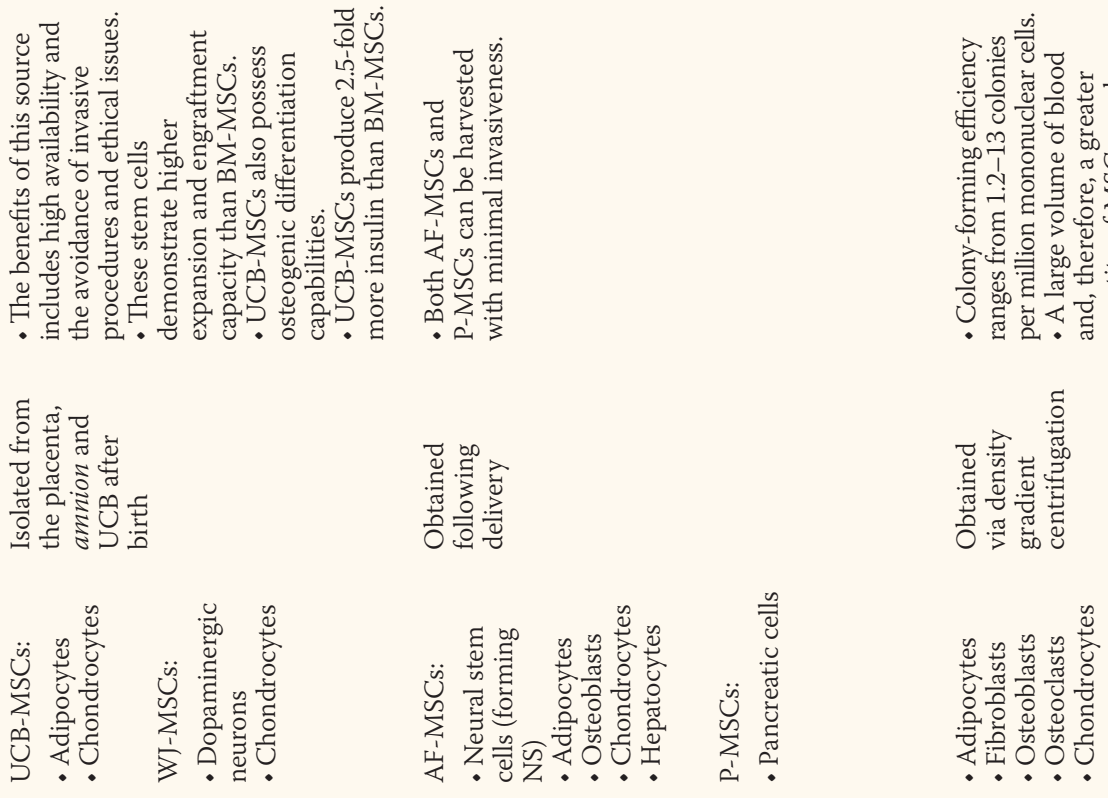

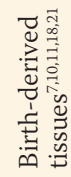

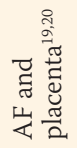

釂 


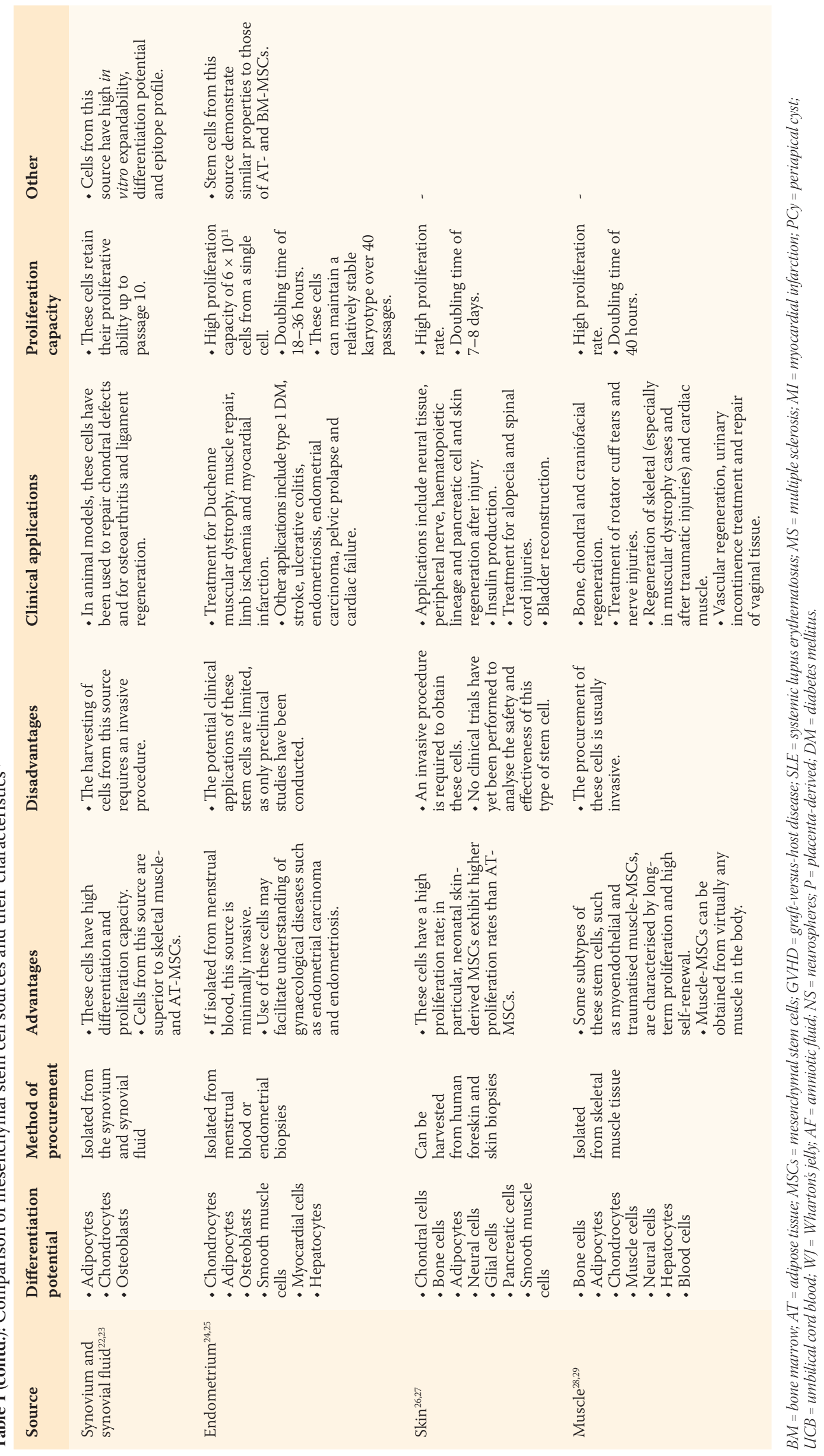


myocyte loss to improve heart function and prevent endstage heart failure. Although clinical trials in both human and animal models have been promising, a meta-analysis of 10 large-scale randomised phase two trials using BMderived cells showed negligible benefits after an acute myocardial infarction. ${ }^{35-42}$ In preclinical and clinical studies, intravenous, intracoronary or intramyocardial routes represent the main options for cell delivery within minutes to a few months after a myocardial infarction..$^{37-42}$ However, protocol variations impede the accurate interpretation of preclinical and clinical trial results. ${ }^{41,42}$ Forest et al. compared cellular retention and engraftment with in vivo imaging in a porcine model using different administration routes. ${ }^{40}$ Following an intracoronary injection, the safest and easiest delivery method, $34.8 \pm 9.9 \%$ of cells were detected in the heart after one hour; this proportion declined precipitously to $6.0 \pm 1.7 \%$ after 24 hours. $^{40}$ Inconsistent results regarding the benefits of BM cell therapy in cardiac function may be due to variability in cell survival, engraftment and differentiation. ${ }^{39}$

Currently, several ongoing clinical trials are being conducted to establish the usefulness of BM-MSCs, especially in the context of ischaemic heart failure. Diastolic left ventricular (LV) dysfunction represents a frequent complication after acute myocardial infarction and a major risk factor for the development of heart failure, independent of preserved LV ejection fraction. ${ }^{41}$ Schaefer et al. conducted a follow-up study of 60 patients with ST-segment elevation acute myocardial infarction treated by BM-MSC transfer. ${ }^{42}$ The time trend of the diastolic function and the E/A ratio remained unchanged in the experimental BM-MSC group relative to the control group, in which the E/A ratio decreased over the course of 60 months. However, the duration of the improvement noted in the experimental group did not exceed five years. ${ }^{42}$ Importantly, no arrhythmogenic or tumourigenic effects have been observed in the treatment of myocardial infarction with BM-MSCs. ${ }^{7}$

In allogeneic (i.e. haematopoietic) stem cell transplantation, graft-versus-host disease (GVHD) - in which immunocompetent donor cells recognise and attack tissues in an immunocompromised host-is a significant cause of morbidity and mortality. ${ }^{11}$ Traditional management entails immunosuppression with corticosteroids as first-line therapy, a treatment that produces sustained responses in $<40-50 \%$ of patients with GVHD. ${ }^{43}$ However, corticosteroid-refractory patients require effective second- and third-line treatment options. ${ }^{44}$ The allogeneic transplantation of BM-MSCs can, when obtained from major histocompatibility complex-matched donors (i.e. a mother or twin), induce immunosuppression in the recipient and thus be used to treat GVHD. This effect was initially evaluated in murine models wherein MSCs inhibited naive and memory antigen-specific T cells. ${ }^{45}$ BM-MSCs have also been used to treat systemic lupus erythematosus (SLE), multiple sclerosis (MS), autoimmune encephalomyelitis, asthma, allergic rhinitis and pulmonary fibrosis. Except for SLE and MS, these applications have all been tested in animal models. ${ }^{7}$ The efficacy and safety of using BM-MSCs in GVDH patients is being tested in ongoing clinical trials based on growing evidence highlighting the key role of BM-MSCs in modulating inflammation. ${ }^{46}$

In the digestive system, BM-MSCs can improve liver cirrhosis and liver failure caused by hepatitis B infections, with positive changes according to chronic liver disease staging and prognostic scales. ${ }^{47}$ Other important applications of BM-MSCs are in orthopaedics. For example, BM aspirates from the iliac crest have been used to treat atrophic diaphyseal non-unions, leading to callus formation. ${ }^{9,12}$ Placing BM-MSCs on scaffolds has been shown to regenerate meniscus tissue in rabbits and BM-MSCs placed on conduits or grafts have been used to treat nerve defects, with better results than cell-depleted grafts. ${ }^{9}$ Furthermore, BM-MSCs have been transplanted to various injured tendon sites, resulting in enhanced tissue repair, especially for injuries of the patellar tendon. ${ }^{9}$

Another important application of BM-MSCs is in the treatment of spinal cord injuries. When mixed with minocycline, BM-MSCs have resulted in improved spinal cord injuries in a rat model. ${ }^{9}$ As such, these cells may represent a promising approach for neuroprotection following spinal cord injury. BM-MSCs have also been used to treat osteogenesis imperfecta in a mouse model, in which BM-MSCs from a diseasefree mouse were infused into an affected mouse. ${ }^{9}$ This treatment promoted the differentiation of migrated cells into osteocytes and synthesised standard amounts of type I collagen, partially reverting the disease phenotype. Other BM-MSC applications include muscular dystrophy, physical defects and rotator cuff injuries. ${ }^{9}$

\section{ADIPOSE TISSUE}

The main advantage of using AT as an MSC source is convenience, as human subcutaneous AT is usually abundant throughout the body and is a byproduct of cosmetic and therapeutic liposuction procedures. It is estimated that approximately $98-100 \%$ of cells obtained from AT are viable. ${ }^{5,48}$ The morphological, phenotypical and functional characteristics of AT-MSCs are similar to those of BM-MSCs. ${ }^{48}$ Besides their stability in longterm cell cultures, AT-MSCs can expand effectively in vitro and possess high multilineage differentiation potential. The stromal vascular AT fraction produces more stromal elements than the mononuclear BM fraction, 
even though these stromal elements may represent other lineages, such as endothelial, haematopoietic and pericyclic origins. ${ }^{48-50}$ Thus, AT represents a more practical autologous source of MSCs for tissue engineering than BM. However, one limitation of AT-MSCs is that certain donor characteristics, such as age, can affect the expansion and differentiation of AT-MSCs, particularly into osteogenic and chondrogenic lineages, although the adipogenic lineage is not affected. ${ }^{48}$

Besides their ability to differentiate and self-renew, AT-MSCs secrete several cytokines and growth factors with anti-inflammatory, antiapoptotic and immunomodulatory properties. Examples include vascular endothelial growth factor, hepatocyte growth factor and insulin-like growth factor, all of which are involved in angiogenesis and tissue repair. These characteristics render AT-MSCs prime candidates for treating ischaemic diseases. ${ }^{5}$ Additionally, due to the immunomodulatory effects of human AT-MSCs, AT is an excellent source of MSCs for allogeneic transplants and, as AT-MSCs do not express major histocompatibility complex type II antigens, the risk of rejection is minimised. ${ }^{11}$ ATMSCs regulate the function of $\mathrm{T}$ cells by promoting the induction of suppressor $\mathrm{T}$ cells and inhibiting the production of cytotoxic $\mathrm{T}$ cells, natural killer cells and proinflammatory cytokines (including tumour necrosis factor- $\alpha$, interferon- $\gamma$ and interleukin [IL]-12). Furthermore, these cells secrete soluble factors, such as IL-10, transforming growth factor- $\beta$ and prostaglandin E2, making these cells immunosuppressive. ${ }^{5,11}$

It should be noted that the $\mathrm{p} 53$ protein plays a key role in the differentiation of human AT-MSCs cells, affecting their osteogenic and adipogenic potential by suppressing the osterix, runt-related transcription factor and peroxisome proliferator-activated receptor (PPAR) genes. ${ }^{51}$ In various malignancies, p53 function is limited, thus offering the possibility of using cancer cells from mutant MSCs to investigate treatment targets. ${ }^{51,52}$ A recent study by Inatani et al. compared the differentiation potential of MSCs from normal AT and atypical lipomatous tumour/well-differentiated liposarcoma (ALT/WDL). ${ }^{49}$ The osteogenic differentiation potential in ALT/WDL-MSCs was greater than that of the normal AT group, although normal AT produced more fatty acid droplets during adipogenic differentiation than ALT/WDL. ${ }^{49}$ This type of study offers an interesting approach to therapeutics in that it postulates using differentiation as a means of treatment. This concept has also been explored with the use of troglitazone in patients with liposarcoma, defective proliferation, apoptosis and differentiation. ${ }^{50}$ Histological tumour changes were observed in a small follow-up study of patients with myxoid and pleomorphic liposarcomas; six months after the administration of PPAR- $\gamma$ ligand, troglitazone, the researchers observed microvesicular cytoplasmic lipid accumulation and diminished proliferation according to Ki-67 expression. ${ }^{50}$ Although imaging indicated that the tumour size increased as a result of intracellular lipid accumulation, with a corresponding increase in cellular volume, no new tumour sites were found. Thus, it may be possible to modify the pathophysiology of cancer and reduce the proliferation of malignant cells by promoting cellular differentiation. ${ }^{50}$

Additionally, AT-MSCs may be useful for myocardial regeneration. In one trial, AT-MSC application resulted in improved cardiac function, elevated perfusion and a reduction in the extension of scar tissue. ${ }^{7}$ AT-MSCs have also been used extensively for dermatological and cosmetic purposes. These cells promote wound healing by enhancing the secretion of type I collagen, upregulating the expression of type III collagen and fibronectin and downregulating matrix metalloproteinase- 1 in fibroblasts. ${ }^{13}$ They have also been shown to accelerate granulation tissue and capillary formation and epithelisation in DM-induced tissue damage. Moreover, AT-MSCs have been used for wrinkle reduction, skin whitening (by inhibiting melanin synthesis), as antioxidants and for the promotion of hair growth. ${ }^{13}$

Furthermore, AT-MSCs have accelerated skeletal muscle regeneration in a rat model..$^{9}$ Interestingly, ATMSCs express various neuronal and Schwann cell markers, such as myelin protein zero, myelin basic protein and peripheral myelin 22, suggesting that they may possess a myelin-generating capacity. These cells have also been used to treat nerve defects and neuropathic pain in rats with promising results. ${ }^{9}$ Finally, AT-MSCs have been used to treat collagenase-induced tendinitis in horses, improving tendon organisation and successfully treating rotator cuff injuries. ${ }^{9}$

\section{DENTAL PULP}

Dental pulp (DP)-derived MSCs specialise into odontoblasts, which produce dentin. ${ }^{14}$ These cells are obtained from pulp tissue from the third molar and are extracted by an enzymatic digestive process. Overall, DP-MSCs are easy to cryopreserve and, similarly to AT-MSCs, possess immunomodulatory properties. ${ }^{14}$ Since DPMSCs are derived from the neural crest, they have ectomesenchymal origins and both ectodermal and mesenchymal lineages. Hence, in addition to differentiating into osteo/chondroblasts and adipocytes, DPMSCs can also differentiate into neuronal lineages. When cultured in a three-dimensional (3D) dentin scaffold, DP-MSCs can differentiate into corneal epithelial cells, melanocytes and induced pluripotent stem cells, rendering them highly useful in regenerative medicine research. ${ }^{14,15,53}$ 
The most promising clinical applications for this stem cell source involve the correction of metabolic diseases and the treatment of liver diseases with high mortality rates, such as cirrhosis and hepatocellular carcinoma. ${ }^{54,55}$ Over time, DP-MSCs have become the preferred alternative to harvesting stem cells during hepatic transplantation. ${ }^{15}$ DP-MSCs have also been used in the field of bone regeneration. When DP-MSCs are cultured on hydrogels, they can spontaneously differentiate into both odontogenic and osteogenic phenotypes. ${ }^{55,56}$

A specific type of DP-MSCs, known as periapical cyst (PCy)-MSCs, have gained special attention because of their extensive proliferative potential, cell surface marker profile and ability to differentiate into various cell types, such as osteoblasts, adipocytes and neurons. ${ }^{16,17,55,56}$ Importantly, these cells are easily collected from surgically removed PCys, thus allowing for the reuse of biological waste. They have also been used in the field of bone regeneration. Due to their neural plasticity, these cells represent an interesting option for treating neurodegenerative diseases. ${ }^{16}$

\section{BIRTH-DERIVED TISSUES}

Currently, umbilical cord blood (UCB) is not universally accepted as a source of MSCs. ${ }^{57}$ Nevertheless, the vast abundance of UCB, availability of donors, ease of procurement and reliability of sample collection and lack of transmission of Herpesviridae viruses are advantages of this particular cell source. ${ }^{57}$ Furthermore, as UCBMSCs are less mature than other types of adult stem cells, they do not elicit a strong immune rejection response in an allogeneic recipient, tolerating mismatches of HLAs. Conveniently, UCB-MSCs can be cryopreserved in vast quantities for later cultivation and research..$^{57}$ The doubling time of these cells is similar to that of BM-MSCs (i.e. every 30 hours). Overall, UCB-MSCs possess osteogenic, chondrogenic, adipogenic and myogenic differentiation potential..$^{57}$ In humans, the intravenous administration of UCB-MSCs has been successfully used to treat GVHD and SLE. ${ }^{11}$ Additionally, UCBMSCs have been implanted to treat sciatic nerve defects and in post-crush and transection animal models with good results. ${ }^{9}$

Recently, Wharton's jelly (WJ) has garnered attention as a potential source of MSCs. The properties of WJMSCs are different from those derived from other sources, with WJ-MSCs exhibiting a higher proliferative potential independent of culture conditions. ${ }^{18}$ Nevertheless, when cultured in the presence of serum, these cells produce fewer extracellular matrix components than BM-MSCs and AT-MSCs. In terms of clinical applications, WJ-MSCs have been shown to be beneficial and safe in the treatment of myocardial infarctions, positively affecting the size of the infarction and $\mathrm{LV}$ function. ${ }^{7}$ In one study, human WJ-MSCs were placed on a 3D scaffold in a conditioned medium obtained from a culture of human articular chondrocytes. ${ }^{18}$ The WJMSCs underwent chondrogenic differentiation without the need for growth factors, with a high accumulation of glycosaminoglycans and expression of cartilage-related genes. These observations indicate that WJ-MSCs may be a good option for regenerating articular cartilage. ${ }^{18}$

\section{AMNIOTIC FLUID AND PLACENTA}

According to immunophenotypical analyses, the phenotype of cultured cells obtained from amniotic fluid (AF) is similar to that of BM-MSCs. ${ }^{58}$ Cultured cells obtained from this source can differentiate into mesenchymal lineages. The expansion of AF- and amnion-derived MSCs is similar, with no marked differences in cell counts, although the latter grow at a slower pace and with a lower yield than BM-MSCs. ${ }^{19,58}$ AF-MSCs demonstrate high self-renewal capacity ( $>300$ cell divisions) and a doubling time of 36 hours. Furthermore, they have been shown to maintain a normal karyotype even at late passages. ${ }^{58}$ Interestingly, AF-MSCs express octamer-binding transcription factor-4 (Oct-4) and stage-specific embryonic antigen-4 (SSEA-4) human embryonic stem cell markers, which is typical for underdifferentiated embryonic stem cells; this means that AF-MSCs are not primitive embryonic stem cells but maintain greater differentiation potential than most adult stem cells. ${ }^{19,58}$

In addition to common mesodermal lineages, AF-MSCs can also differentiate into hepatocytes and neurons under specific culture conditions. ${ }^{19}$ While no human investigations using AF-MSCs have yet been reported, several animal models have studied various clinical applications, including bladder formation using AF-MSCs differentiated into muscle cells, the treatment of nerve injuries, blood vessel and heart valve formation and regeneration of the diaphragm, kidney, bone, lung, heart and cartilage. 19,20,58 As with UCB-MSCs, AF-MSCs have also been successfully implanted in sciatic nerve defects and post-crush and transection animal models. ${ }^{9}$

The basement membrane of the amnion contains MSCs derived from the inner cell mass of blastocysts. In addition to not expressing HLA class II, human amnionderived MSCs possess immunosuppressive factors. ${ }^{58}$ Other parts of the placenta, such as the decidua basalis and decidua parietalis, also contain MSCs; upon cultivation, these cells exhibit similar characteristics to amnionderived MSCs. Placenta-derived MSCs (P-MSCs) also express embryonic stem cell markers such as c-Kit, sex determining region Y-box 2, Oct- 4 and SSEA-4 and are able to differentiate into mesenchymal as well 
as hepatic, pancreatic and neuronal lineages. ${ }^{59}$ P-MSCs have been shown to improve lung function and reduce pulmonary fibrosis in animal models and have been used in the treatment of skin and ocular diseases..$^{20,59}$ Interestingly, these cells have also been used to create an artificial amnion within a collagen scaffold. ${ }^{19}$ Other clinical applications of P-MSCs in animal models include the formation of cardiac tissue and treatment of bleomycin-induced lung injuries, cartilage defects, inflammatory bowel disease, Duchenne muscular dystrophy, ischaemic stroke, inflammation and DM. ${ }^{20}$ One of the main benefits of P-MSCs is that they can be easily obtained following delivery without the need for a specific procedure; they also offer distinct advantages in terms of proliferation and plasticity. ${ }^{20}$

\section{MOBILISED PERIPHERAL BLOOD}

Specific factors that control haematopoiesis were initially identified by their ability to support progenitor cells in culture assays. These factors have since been isolated and purified, allowing for the development of therapeutic agents, including colony-stimulating factors (CSFs). ${ }^{60,61}$ Filgrastim, a granulocyte-CSF, is a bacterially-synthesised recombinant protein that acts on neutrophils. It was first used in cancer patients in 1991 as an adjunct for ameliorating chemotherapy-induced neutropaenia. Filgrastim has since been approved in over 70 countries for mobilising peripheral blood progenitor cells for transplantation. ${ }^{60,61}$ Granulocyte-CSF binds selectively to specific cell-surface receptors, triggering the phosphorylation of janus kinase (JAK) 1 and JAK2 protein tyrosine kinases and activating the Ras/mitogen-activated protein kinase pathway. A subcutaneous dose of filgrastim $(10 \mu \mathrm{g} / \mathrm{kg})$ results in a six-fold increase in neutrophil levels within 24-48 hours. ${ }^{62}$ Furthermore, filgrastim treatment reduces the neutrophil maturation time from five days to one day. ${ }^{61,62}$

Peripheral blood stem cells may be mobilised from healthy donors using granulocyte-CSFs. ${ }^{9}$ The underlying mechanism involves a number of adhesion molecules, including lymphocyte function-associated antigen 1, very late antigen-4, C-X-C motif chemokine receptor 4, c-Kit, CD44 and macrophage-1. These molecules facilitate the binding of stem cells to BM and their disruption allows for their release into the circulation. While the underlying mechanism is unclear, this process is mainly regulated by cytokines. ${ }^{63,64}$

As a replacement for BM-MSCs, granulocyteCSF-mobilised peripheral blood stem cells are convenient and easily accessible. They are similar to BMMSCs in most aspects, as they can differentiate into mesenchymal lineages and have a strong capacity to adhere to plastic culture flasks. ${ }^{21}$ However, MSCs mobilised from the blood using granulocyte-CSF exhibit a 95-hour duplication time, which is longer than that of MSCs from other sources. Moreover, they adhere in a monolayer and to different scaffolds. ${ }^{21}$ Their surface expression profile is similar to that of BM-MSCs with specific MSC markers. Their capability to differentiate into chondral and bone lineages is lower than that of BM-MSCs, while their capability to differentiate into an adipose lineage is higher. ${ }^{21}$ As yet, no clinical trial on the use of these cells has been published.

\section{SYNOVIUM AND SYNOVIAL FLUID}

In humans, cartilage and synovium originate from a common pool of cells during synovial joint development. In the treatment of musculoskeletal injuries, synovial MSCs are an attractive method of meniscus regeneration. Synovium-derived MSCs (S-MSCs) are far superior to cells derived from the skeletal muscle and AT, as determined by their in vitro expandability, differentiation potential and epitope profiles. ${ }^{65}$ When replated at a density of 50 cells $/ \mathrm{cm}^{2}$, these cells retain their proliferative ability up to passage 10 , whereas cells from other sources proliferate only until passage seven. Furthermore, these cells possess remarkable adipogenic, chondrogenic and osteogenic potential comparable to that of BM- and periosteum-derived MSCs. ${ }^{65}$ Interestingly, in vivo evidence suggests that the intra-articular injection of endogenous cells from the synovial membrane can contribute to the repair of partial-thickness cartilage defects in a mouse injury model. ${ }^{22} \mathrm{~S}$-MSCs have also promoted ligament regeneration in another animal model. ${ }^{23}$

Interestingly, in one study, when S-MSCs were implanted into the deeper aspect of an osteochondral defect, they differentiated into osteocytes; however, when they were implanted into the superficial aspect, the cells differentiated into chondrocytes. ${ }^{9}$ These observations suggest that the microenvironment may play an important role in the multilineage differentiation potential of MSCs. ${ }^{9}$ Furthermore, S-MSCs may play a role in the endogenous repair of intra-articular injuries because the number of S-MSCs in synovial fluid are elevated in patients with osteoarthritic knees, cartilage defects, meniscus damage or intra-articular ligament injuries. ${ }^{9}$

\section{ENDOMETRIUM}

In 2004, Chan et al. hypothesised that because the human endometrium regenerates from the lower basalis layer, it might contain small populations of epithelial and stromal cells that exhibit clonogenicity ${ }^{66}$ Human clonogenic endometrial cells exhibit the same in vitro properties as BM-MSCs. Human endometrial stromal cell cultures can differentiate in vitro, unlike other nonendometrial gynaecological tissues. ${ }^{67,68}$ 
There are three types of endometrial stem cells (EnSCs): epithelial progenitor cells, MSCs and endothelial progenitor cells. ${ }^{68}$ EnSCs can be isolated from the menstrual blood or from endometrial biopsies without the need for invasive procedures. ${ }^{68,69}$ Furthermore, EnSCs have similar properties to those of AT- and BMderived MSCs. The functional dysregulation of these cells may contribute to a range of pathologies, from endometriosis to endometrial carcinomas. ${ }^{24}$ The proliferation potential of EnSCs is $6 \times 10^{11}$ cells from a single cell and these cells can differentiate into adipogenic, chondrogenic and osteogenic lineages. ${ }^{67,68}$ EnSCs have several clinical applications, albeit limited to preclinical studies. Current models include Duchenne muscular dystrophy, muscle repair by EnSC transplantation into atrophied muscle fibres, limb ischaemia and myocardial infarction. ${ }^{68}$

The proliferative ability of menstrual blood-derived MSCs (Men-MSCs) is very high and they can maintain a relatively stable karyotype over 40 passages. The doubling time of these cells is 18-36 hours, which is fast when compared with other types of MSCs. ${ }^{69-71}$ Men-MSCs can be induced into multiple lineages, including chondrocytes, osteoblasts, adipocytes, smooth muscle cells, myocardial cells and hepatocytes. In an animal model of type $1 \mathrm{DM}$, the intravenous administration of these cells improved hyperglycaemia. ${ }^{72}$ In another animal model, Men-MSCs were shown to secrete various neuroprotective factors (e.g. vascular endothelial growth factor and brain-derived neurotrophic factor) which promote neuron survival and mitigate behavioural and histological changes, making them applicable for stroke treatment. ${ }^{25}$ Other potential applications of Men-MSCs include ulcerative colitis, endometriosis, endometrial carcinomas, pelvic organ prolapse and cardiac failure. ${ }^{25}$

\section{SKIN}

Skin-derived precursors are able to differentiate into both neural and mesodermal cells. These cells can be harvested from the human foreskin or even from skin biopsies. ${ }^{73}$ When cultured, they differentiate into neurons, glia and smooth muscle cells, including cells of the peripheral neural phenotype (consistent with cells originating from the neural crest), but hold limited potential for mesenchymal lineage differentiation. ${ }^{26,73}$ One of the main advantages of this cell type is its proliferation rate. The skinderived MSCs (S-MSCs) of human newborns exhibit a higher proliferation rate during long-term cultures than human AT-MSCs and skin stromal cells. ${ }^{68,73}$ Furthermore, stem cells can be obtained from several regions of the skin, including the epidermis (epidermal stem cells), interfollicular epidermis, hair follicles, sebaceous glands and subcutaneous AT. Each of these stem cell types expresses different markers. ${ }^{26,27}$
Although S-MSCs are able to differentiate into neurons, from a functional point of view, these cells present an immature electrophysiological profile. ${ }^{27}$ The implantation of S-MSCs into an adult mouse brain led to the discovery that these cells are capable of differentiating into astrocytes as well as insulinproducing pancreatic cells. ${ }^{27}$ An important application of S-MSCs is skin regeneration after serious skin damage, such as that caused by burns, chronic ulcers and deep wounds, as well as anti-ageing therapy and repigmentation in vitiligo, alopaecia and melanoma cases. Other potential clinical applications of S-MSCs currently being studied include spinal cord injuries, regeneration of the haematopoietic lineage after radiotherapy, bladder reconstruction and alopecia. ${ }^{27}$ Undifferentiated hair follicle stem cells have also been used in murine models of sciatic and tibial nerve transections and crush injuries, with the animals exhibiting improved functional outcomes after cell administration. ${ }^{9}$

\section{MUSCLE}

The skeletal muscle and bone share a common mesodermal origin and these tissues exhibit regenerative capacities due to the presence of endogenous muscle stem cells, satellite cells and skeletal stem cells. ${ }^{74}$ It is important to distinguish between satellite cells and muscle-derived MSCs (M-MSCs), as the former are committed to a myogenic lineage while the latter are satellite cell predecessors capable of multilineage differentiation. Other subtypes of M-MSCs include myoendothelial cells-which express surface markers of both endothelial and satellite cells-and traumatised M-MSCs which are rapidly adherent. ${ }^{74}$ M-MSCs differentiate into mesenchymal tissues, including osteogenic, myogenic and even chondrogenic lineages. The most important and extensively researched clinical application of this cell type is for use in the treatment of muscular dystrophy. ${ }^{75}$

Overall, M-MSCs have many clinical applications; for instance, upon transfection with the bone morphogenetic protein-4 gene, M-MSCs were able to differentiate into osteoblasts and fill a critical femoral defect in an animal model. ${ }^{28}$ These cells have also been used in a similar fashion for craniofacial regeneration. In an animal model, the implantation of M-MSCs with fibrin glue directly into osteochondral defects resulted in persistent repair of the defect site after 24 weeks. ${ }^{29}$ These cells, when differentiated, express type II collagen and proteoglycan-rich extracellular matrix.

M-MSCs may also promote peripheral nerve regeneration, as shown in animal models of sciatic nerve injuries. ${ }^{9}$ Another therapeutic application of M-MSCs is the promotion of skeletal and cardiac muscle regen- 
eration. Several studies have supported the possibility that M-MSCs can augment muscle healing following injury; another study found that the injection of M-MSCs into the infarcted region of cardiac tissue resulted in improved function..$^{28}$ Additionally, M-MSCs can mediate a secondary mechanism of regeneration by promoting angiogenesis. ${ }^{28,30}$ Other potential applications of M-MSCs include vascular regeneration, the treatment of urinary incontinence and the repair of vaginal tissue. M-MSCs have also been successfully used for treating rotator cuff injuries. ${ }^{9}$

\section{Legal Implications and Future Research}

The wide array of possibilities engendered by stem cell therapy have generated expectations that exceed our current understanding and regulations regarding their use. To meet these expectations, the Food and Drug Administration (FDA) in the USA has advocated further studies to assess the risks and benefits of stem cell therapy and develop evidence-based therapeutic approaches. ${ }^{76}$ Thus far, the FDA has only approved stem cell treatments sourced from BM aspirate concentrate. ${ }^{9}$ While haematopoietic reconstitution using BM- or peripheral blood-derived MSCs is a well-known, safe and effective treatment, there is a lack of research regarding the safety and efficacy of other sources of MSCs. In some cases, these sources may be used with only minimal clinical evidence. ${ }^{76-78}$

Some of the arguments used to justify these practices include the capacity of MSCs to repair damage; the extremely high cost of clinical trials necessitating large industrial sponsors; and the assumption that stem cell therapies are safe, especially when derived from an autologous source. ${ }^{76,77}$ The modern clinician should understand the basis of regenerative medicine, the clinical options at their disposal and the potential of such therapies to drive medical decisions. However, they must also protect patients from pseudomedical treatments that have been widely popularised to constitute stem cell therapies. Most clinical options for stem cell therapy focus on chronic conditions because of the relatively slow pace of transplantable stem cell generation. In general, such procedures require the procurement, isolation, expansion, differentiation and implantation of a sufficient number of cells before a therapeutic response is noted. ${ }^{76-78}$

In different countries, regulations for regenerative medicine products allow for exceptions to longestablished safety rules. For example, in Japan, such products can be marketed based on preliminary evid- ence and are only required to meet reduced thresholds for therapeutic efficacy. ${ }^{74}$ In the USA, the $21^{\text {st }}$ Century Cures Act of 2016 saw the inception of the term 'regenerative advanced therapy' for the purpose of expediting regulatory processes. ${ }^{77}$ Nevertheless, clinical trials should be performed to ensure the safe and effective use of therapies involving MSCs, regardless of cell source or clinical application. Moreover, specific laws should be implemented for further control over the use of these cells. ${ }^{78}$

In the future, once the aforementioned challenges have been resolved, it might be possible to replicate the exact function of native tissues by differentiating MSCs identical to the organs in need of replacement. Furthermore, the differentiation process might be performed in vivo, leading to standardised stem cell therapies for various ailments.

\section{Conclusion}

Although BM-MSCs have been the main source of stem cells for many years, the inconvenience and risk of procuring these cells has fuelled research for alternative and more practical sources. As shown in this review, MSCs have many clinical applications, with an important role in almost all medical and surgical specialties. It is important to recognise that the therapeutic use of MSCs is not an unrealistic goal, as stem cells already offer a promising option in the treatment of several diseases. Nevertheless, the safety and efficacy of all types of MSCs, regardless of source, should be carefully assessed prior to their application, first in animal models and subsequently in clinical trials.

\section{References}

1. Choo A, Ngo AS, Ding V, Oh S, Kiang LS. Autogeneic feeders for the culture of undifferentiated human embryonic stem cells in feeder and feeder-free conditions. Methods Cell Biol 2008; 86:15-28. doi: 10.1016/S0091-679X(08)00002-2.

2. Hiew VV, Simat SFB, Teoh PL. The advancement of biomaterials in regulating stem cell fate. Stem Cell Rev 2018; 14:43-57. doi: 10.1007/s12015-017-9764-y.

3. Lv FJ, Tuan RS, Cheung KM, Leung VY. Concise review: The surface markers and identity of human mesenchymal stem cells. Stem Cells 2014; 32:1408-19. doi: 10.1002/stem.1681.

4. Wei X, Yang X, Han ZP, Qu FF, Shao L, Shi YF. Mesenchymal stem cells: A new trend for cell therapy. Acta Pharmacol Sin 2013; 34:747-54. doi: 10.1038/aps.2013.50.

5. Liras A. Future research and therapeutic applications of human stem cells: General, regulatory, and bioethical aspects. J Transl Med 2010; 8:131. doi: 10.1186/1479-5876-8-131.

6. Stanko P, Kaiserova K, Altanerova V, Altaner C. Comparison of human mesenchymal stem cells derived from dental pulp, bone marrow, adipose tissue, and umbilical cord tissue by gene expression. Biomed Pap Med Fac Univ Palacky Olomouc Czech Repub 2014; 158:373-7. doi: 10.5507/bp.2013.078. 
7. Majka M, Sułkowski M, Badyra B, Musiałek P. Concise review: Mesenchymal stem cells in cardiovascular regeneration - Emerging research directions and clinical applications. Stem Cells Transl Med 2017; 6:1859-67. doi: 10.1002/sctm.16-0484.

8. Dominici M, Le Blanc K, Mueller I, Slaper-Cortenbach I, Marini F, Krause D, et al. Minimal criteria for defining multipotent mesenchymal stromal cells: The International Society for Cellular Therapy position statement. Cytotherapy 2006; 8:315-17. doi: 10.1080/14653240600855905.

9. Berebichez-Fridman R, Gómez-García R, Granados-Montiel J, Berebichez-Fastlicht E, Olivos-Meza A, Granados J, et al. The holy grail of orthopedic surgery: Mesenchymal stem cells - Their current uses and potential applications. Stem Cells Int 2017; 2017:2638305. doi: $10.1155 / 2017 / 2638305$

10. Cheng HY, Ghetu N, Wallace CG, Wei FC, Liao SK. The impact of mesenchymal stem cell source on proliferation, differentiation, immunomodulation and therapeutic efficacy. J Stem Cell Res Ther 2014; 4:237. doi: 10.4172/2157-7633.1000237.

11. Cagliani J, Grande D, Molmenti EP, Miller EJ, Rilo HLR Immunomodulation by mesenchymal stromal cells and their clinical applications. J Stem Cell Regen Biol 2017; 3:1-26. doi: 10.15436/2471-0598.17.022.

12. Mafi R, Hindocha S, Mafi P, Griffin M, Khan WS. Sources of adult mesenchymal stem cells applicable for musculoskeletal applications: A systematic review of the literature. Open Orthop J 2011; 5:242-8. doi: 10.2174/1874325001105010242.

13. Yang JA, Chung HM, Won CH, Sung JH. Potential application of adipose-derived stem cells and their secretory factors to skin: Discussion from both clinical and industrial viewpoints. Expert Opin Biol Ther 2010; 10:495-503. doi: 10.1517/14712591003610598.

14. Gronthos S, Mankani M, Brahim J, Robey PG, Shi S. Postnatal human dental pulp stem cells (DPSCs) in vitro and in vivo. Proc Natl Acad Sci U S A 2000; 97:13625-30. doi: 10.1073/pnas.240 309797.

15. Kushnerev E, Shawcross SG, Sothirachagan S, Carley F, Brahma A Yates JM, et al. Regeneration of corneal epithelium with dental pulp stem cells using a contact lens delivery system. Invest Ophthalmol Vis Sci 2016; 57:5192-9. doi: 10.1167/iovs.15-17953.

16. Tatullo M, Codispoti B, Pacifici A, Palmieri F, Marrelli M, Pacifici L, et al. Potential use of human periapical cyst-mesenchymal stem cells (hPCy-MSCs) as a novel stem cell source for regenerative medicine applications. Front Cell Dev Biol 2017; 5:103. doi: 10.3389/fcell.2017.00103.

17. Vasandan AB, Shankar SR, Prasad P, Sowmya Jahnavi V, Bhonde RR, Jyothi Prasanna S. Functional differences in mesenchymal stromal cells from human dental pulp and periodontal ligament. J Cell Mol Med 2014; 18:344-54. doi: 10.1111/jcmm.12192.

18. Alves da Silva ML, Costa-Pinto AR, Martins A, Correlo VM, Sol P, Bhattacharya $\mathrm{M}$, et al. Conditioned medium as a strategy for human stem cells chondrogenic differentiation. J Tissue Eng Regen Med 2015; 9:714-23. doi: 10.1002/term.1812.

19. Joo S, Ko IK, Atala A, Yoo JJ, Lee SJ. Amniotic fluid-derived stem cells in regenerative medicine research. Arch Pharm Res 2012; 35:271-80. doi: 10.1007/s12272-012-0207-7.

20. Pipino C, Shangaris P, Resca E, Zia S, Deprest J, Sebire NJ, et al. Placenta as a reservoir of stem cells: An underutilized resource? Br Med Bull 2013; 105:43-68. doi: 10.1093/bmb/lds033.

21. Tondreau T, Meuleman N, Delforge A, Dejeneffe M, Leroy R, Massy M, et al. Mesenchymal stem cells derived from CD133positive cells in mobilized peripheral blood and cord blood: Proliferation, Oct4 expression, and plasticity. Stem Cells 2005; 23:1105-12 doi: 10.1634 /stemcells. 2004-0330.

22. Mak J, Jablonski CL, Leonard CA, Dunn JF, Raharjo E, Matyas JR, et al. Intra-articular injection of synovial mesenchymal stem cells improves cartilage repair in a mouse injury model. Sci Rep 2016; 6:23076. doi: 10.1038/srep23076.
23. McGonagle D, Jones E. A potential role for synovial fluid mesenchymal stem cells in ligament regeneration. Rheumatology (Oxford) 2008; 47:1114-16. doi: 10.1093/rheumatology/ken236.

24. Gargett CE, Masuda H. Adult stem cells in the endometrium. Mol Hum Reprod 2010; 16:818-34. doi: 10.1093/molehr/gaq061.

25. Xu Y, Zhu H, Zhao D, Tan J. Endometrial stem cells: Clinical application and pathological roles. Int J Clin Exp Med 2015; 8:22039-44

26. Toma JG, McKenzie IA, Bagli D, Miller FD. Isolation and characterization of multipotent skin-derived precursors from human skin. Stem Cells 2005; 23:727-37. doi: 10.1634/stemcells. 2004-0134.

27. Niezgoda A, Niezgoda P, Nowowiejska L, Białecka A, MęcińskaJundziłł K, Adamska U, et al. Properties of skin stem cells and their potential clinical applications in modern dermatology. Eur J Dermatol 2017; 27:227-36. doi: 10.1684/ejd.2017.2988.

28. Jackson WM, Nesti LJ, Tuan RS. Potential therapeutic applications of muscle-derived mesenchymal stem and progenitor cells. Expert Opin Biol Ther 2010; 10:505-17. doi: 10.1517/147 12591003610606.

29. Gates CB, Karthikeyan T, Fu F, Huard J. Regenerative medicine for the musculoskeletal system based on muscle-derived stem cells. J Am Acad Orthop Surg 2008; 16:68-76.

30. Hass R, Kasper C, Böhm S, Jacobs R. Different populations and sources of human mesenchymal stem cells (MSC): A comparison of adult and neonatal tissue-derived MSC. Cell Commun Signal 2011; 9:12. doi: 10.1186/1478-811X-9-12.

31. Friedenstein AJ. Precursor cells of mechanocytes. Int Rev Cytol 1976; 47:327-59.

32. Friedenstein AJ, Chailakhyan RK, Gerasimov UV. Bone marrow osteogenic stem cells: In vitro cultivation and transplantation in diffusion chambers. Cell Tissue Kinet 1987; 20:263-72.

33. Kern S, Eichler H, Stoeve J, Klüter H, Bieback K. Comparative analysis of mesenchymal stem cells from bone marrow, umbilical cord blood, or adipose tissue. Stem Cells 2006; 24:1294-301. doi: 10.1634/stemcells.2005-0342.

34. Penn MS, Swaminath D. Novel role of bone marrow stem cells in systemic disease. Circ Res 2015; 117:119-20. doi: 10.1161/ CIRCRESAHA.117.306852.

35. Jackson L, Jones DR, Scotting P, Sottile V. Adult mesenchymal stem cells: Differentiation potential and therapeutic applications. J Postgrad Med 2007; 53:121-7. doi: 10.4103/0022-3859.32215.

36. Lipinski MJ, Biondi-Zoccai GG, Abbate A, Khianey R, Sheiban I, Bartunek J, et al. Impact of intracoronary cell therapy on left ventricular function in the setting of acute myocardial infarction: A collaborative systematic review and meta-analysis of controlled clinical trials. J Am Coll Cardiol 2007; 50:1761-7. doi: 10.1016/j.jacc.2007.07.041.

37. Lloyd-Jones D, Adams RJ, Brown TM, Carnethon M, Dai S, De Simone G, et al. Heart disease and stroke statistics: 2010 update - A report from the American Heart Association. Circulation 2010; 121:e46-215. doi: 10.1161/CIRCULATIONAHA. 109.192667 .

38. Segers VF, Lee RT. Stem-cell therapy for cardiac disease. Nature 2008; 451:937-42. doi: 10.1038/nature06800

39. Nguyen PK, Lan F, Wang Y, Wu JC. Imaging: Guiding the clinical translation of cardiac stem cell therapy. Circ Res 2011; 109:962-79. doi: 10.1161/CIRCRESAHA.111.242909.

40. Forest VF, Tirouvanziam AM, Perigaud C, Fernandes S, Fusellier MS, Desfontis JC, et al. Cell distribution after intracoronary bone marrow stem cell delivery in damaged and undamaged myocardium: Implications for clinical trials. Stem Cell Res Ther 2010; 1:4. doi: $10.1186 /$ scrt4. 
41. Poulsen SH, Jensen SE, Egstrup K. Longitudinal changes and prognostic implications of left ventricular diastolic function in first acute myocardial infarction. Am Heart J 1999; 137:910-18. doi: 10.1016/S0002-8703(99)70416-3

42. Schaefer A, Zwadlo C, Fuchs M, Meyer GP, Lippolt P, Wollert KC, et al. Long-term effects of intracoronary bone marrow cell transfer on diastolic function in patients after acute myocardial infarction: 5-year results from the randomized-controlled BOOST trial - An echocardiographic study. Eur J Echocardiogr 2010; 11:165-71. doi: 10.1093/ejechocard/jep191.

43. Saliba RM, de Lima M, Giralt S, Andersson B, Khouri IF, Hosing C, et al. Hyperacute GVHD: Risk factors, outcomes, and clinical implications. Blood 2006; 109:2751-8. doi: 10.1182/ blood-2006-07-034348.

44. Le Blanc K, Rasmusson I, Sundberg B, Götherström C, Hassan M, Uzunel $\mathrm{M}$, et al. Treatment of severe acute graft-versus-host disease with third party haploidentical mesenchymal stem cells. Lancet 2004; 363:1439-41. doi: 10.1016/S0140-6736(04)16104-7.

45. Krampera M, Glennie S, Dyson J, Scott D, Laylor R, Simpson E, et al. Bone marrow mesenchymal stem cells inhibit the response of naive and memory antigen-specific $\mathrm{T}$ cells to their cognate peptide. Blood 2003; 101:3722-9. doi: 10.1182/blood-2002-07-2104.

46. Wang LT, Ting CH, Yen ML, Liu KJ, Sytwu HK, Wu KK, et al. Human mesenchymal stem cells (MSCs) for treatment towards immune- and inflammation-mediated diseases: Review of current clinical trials. J Biomed Sci 2016; 23:76. doi: 10.1186/s12929016-0289-5.

47. Houlihan DD, Hopkins LJ, Suresh SX, Armstrong MJ, Newsome PN Autologous bone marrow mesenchymal stem cell transplantation in liver failure patients caused by hepatitis B: Short-term and longterm outcomes. Hepatology 2011; 54:1891-2. doi: 10.1002/hep.24722.

48. Choudhery MS, Badowski M, Muise A, Pierce J, Harris DT. Donor age negatively impacts adipose tissue-derived mesenchymal stem cell expansion and differentiation. J Transl Med 2014: $12 \cdot 8$ doi: $10.1186 / 1479-5876-12-8$.

49. Inatani H, Yamamoto N, Hayashi K, Kimura H, Takeuchi A, Miwa S, et al. Do mesenchymal stem cells derived from atypical lipomatous tumors have greater differentiation potency than cells from normal adipose tissues? Clin Orthop Relat Res 2017; 475:1693-701. doi: 10.1007/s11999-017-5259-z.

50. Demetri GD, Fletcher CD, Mueller E, Sarraf P, Naujoks R, Campbell $\mathrm{N}$, et al. Induction of solid tumor differentiation by the peroxisome proliferator-activated receptor-gamma ligand troglitazone in patients with liposarcoma. Proc Natl Acad Sci U S A 1999; 96:3951-6. doi: 10.1073/pnas.96.7.3951.

51. Artigas N, Gámez B, Cubillos-Rojas M, Sánchez-de Diego C, Valer JA, Pons G, et al. p53 inhibits SP7/osterix activity in the transcriptional program of osteoblast differentiation. Cell Death Differ 2017; 24:2022-31. doi: 10.1038/cdd.2017.113.

52. Reya T, Morrison SJ, Clarke MF, Weissman IL. Stem cells, cancer, and cancer stem cells. Nature 2001; 414:105-11. doi: $10.1038 / 35102167$

53. Sanz AR, Carrión ES, Chaparro AP. Mesenchymal stem cells from the oral cavity and their potential value in tissue engineering. Periodontol 2000 2015; 67:251-67. doi: 10.1111/prd.12070.

54. Ohkoshi S, Hara H, Hirono H, Watanabe K, Hasegawa K. Regenerative medicine using dental pulp stem cells for liver diseases. World J Gastrointest Pharmacol Ther 2017; 8:1-6. doi: 10.4292/ wjgpt.v8.i1.1.

55. Ishkitiev N, Yaegaki K, Calenic B, Nakahara T, Ishikawa H, Mitiev V, et al. Deciduous and permanent dental pulp mesenchymal cells acquire hepatic morphologic and functional features in vitro. J Endod 2010; 36:469-74. doi: 10.1016/j.joen.2009.12.022.

56. Lei M, Li K, Li B, Gao LN, Chen FM, Jin Y. Mesenchymal stem cell characteristics of dental pulp and periodontal ligament stem cells after in vivo transplantation. Biomaterials 2014; 35:6332-43. doi: 10.1016/j.biomaterials.2014.04.071.
57. Gang EJ, Hong SH, Jeong JA, Hwang SH, Kim SW, Yang IH, et al. In vitro mesengenic potential of human umbilical cord blood-derived mesenchymal stem cells. Biochem Biophys Res Commun 2004; 321:102-8. doi: 10.1016/j.bbrc.2004.06.111.

58. Koike C, Zhou K, Takeda Y, Fathy M, Okabe M, Yoshida T, et al. Characterization of amniotic stem cells. Cell Reprogram 2014; 16:298-305. doi: 10.1089/cell.2013.0090.

59. In't Anker PS, Scherjon SA, Kleijburg-van der Keur C, de Groot-Swings GM, Claas FH, Fibbe WE, et al. Isolation of mesenchymal stem cells of fetal or maternal origin from human placenta. Stem Cells 2004; 22:1338-45. doi: 10.1634/ stemcells.2004-0058

60. Demetri GD, Griffin JD. Granulocyte colony-stimulating factor and its receptor. Blood 1991; 78:2791-808.

61. Chao NJ, Schriber JR, Grimes K, Long GD, Negrin RS, Raimondi CM, et al. Granulocyte colony-stimulating factor "mobilized" peripheral blood progenitor cells accelerate granulocyte and platelet recovery after high-dose chemotherapy. Blood 1993; 81:2031-5.

62. Welte K, Gabrilove J, Bronchud MH, Platzer E, Morstyn G. Filgrastim (r-metHuG-CSF): The first 10 years. Blood 1996; 88:1907-29.

63. Craddock CF, Nakamoto B, Andrews RG, Priestley GV, Papayannopoulou T. Antibodies to VLA4 integrin mobilize long-term repopulating cells and augment cytokine-induced mobilization in primates and mice. Blood 1997; 90:4779-88.

64. Kikuta T, Shimazaki C, Ashihara E, Sudo Y, Hirai H, Sumikuma T, et al. Mobilization of hematopoietic primitive and committed progenitor cells into blood in mice by anti-vascular adhesion molecule-1 antibody alone or in combination with granulocyte colony-stimulating factor. Exp Hematol 2000; 28:311-17. doi: 10.1016/S0301-472X(99)00151-4.

65. Sakaguchi Y, Sekiya I, Yagishita K, Muneta T. Comparison of human stem cells derived from various mesenchymal tissues: Superiority of synovium as a cell source. Arthritis Rheum 2005; 52:2521-9. doi: $10.1002 /$ art.21212

66. Chan RW, Schwab KE, Gargett CE. Clonogenicity of human endometrial epithelial and stromal cells. Biol Reprod 2004; 70:1738-50. doi: 10.1095/biolreprod.103.024109.

67. Wolff EF, Wolff AB, Hongling Du, Taylor HS. Demonstration of multipotent stem cells in the adult human endometrium by in vitro chondrogenesis. Reprod Sci 2007; 14:524-33. doi: 10.1177/ 1933719107306896.

68. Verdi J, Tan A, Shoae-Hassani A, Seifalian AM. Endometrial stem cells in regenerative medicine. J Biol Eng 2014; 8:20. doi: 10.1186/1754-1611-8-20.

69. Gargett CE. Uterine stem cells: What is the evidence? Hum Reprod Update 2007; 13:87-101. doi: 10.1093/humupd/dml045.

70. Schüring AN, Schulte N, Kelsch R, Röpke A, Kiesel L, Götte M. Characterization of endometrial mesenchymal stem-like cells obtained by endometrial biopsy during routine diagnostics. Fertil Steril 2011; 95:423-6. doi: 10.1016/j.fertnstert.2010.08.035.

71. Pittenger MF, Mackay AM, Beck SC, Jaiswal RK, Douglas R, Mosca JD, et al. Multilineage potential of adult human mesenchymal stem cells. Science 1999; 284:143-7. doi: 10.1126/science. 284.5411.143.

72. Wu X, Luo Y, Chen J, Pan R, Xiang B, Du X, et al. Transplantation of human menstrual blood progenitor cells improves hyperglycemia by promoting endogenous progenitor differentiation in type 1 diabetic mice. Stem Cells Dev 2014; 23:124557. doi: $10.1089 /$ scd.2013.0390.

73. Al-Nbaheen M, Vishnubalaji R, Ali D, Bouslimi A, Al-Jassir F, Megges M, et al. Human stromal (mesenchymal) stem cells from bone marrow, adipose tissue and skin exhibit differences in molecular phenotype and differentiation potential. Stem Cell Rev 2013; 9:32-43. doi: 10.1007/s12015-012-9365-8. 
74. Abou-Khalil R, Yang F, Lieu S, Julien A, Perry J, Pereira C, et al. Role of muscle stem cells during skeletal regeneration. Stem Cells 2015; 33:1501-11. doi: 10.1002/stem.1945.

75. Peng H, Huard J. Muscle-derived stem cells for musculoskeletal tissue regeneration and repair. Transpl Immunol 2004; 12:311-19. doi: 10.1016/j.trim.2003.12.009.
76. Marks PW, Witten CM, Califf RM. Clarifying stem-cell therapy's benefits and risks. N Engl J Med 2017; 376:1007-9. doi: 10.1056/NEJMp1613723

77. Daley GQ. Polar extremes in the clinical use of stem cells. N Engl J Med 2017; 376:1075-7. doi: 10.1056/NEJMe1701379.

78. Marks P, Gottlieb S. Balancing safety and innovation for cellbased regenerative medicine. N Engl J Med 2018; 378:954-9. doi: 10.1056/NEJMsr1715626. 